\title{
A COMPREHENISVE ANALYSIS OF BENCHMARK 4: PRE-STRAIN EFFECT ON SPRINGBACK OF 2D DRAW BENDING
}

\author{
P.-A. Eggertsen ${ }^{\mathrm{a}, \mathrm{b}}$, Kjell Mattiasson ${ }^{\mathrm{a}, \mathrm{b}}$, Mats Larsson ${ }^{\mathrm{c}, \mathrm{d}}$ \\ ${ }^{a}$ Div. of Material and Computational Mechanics, Dept. of Applied Mechanics \\ Chalmers University of Technology, SE-412 96 Göteborg, Sweden \\ ${ }^{b}$ Volvo Cars Safety Centre, SE-405 31 Göteborg, Sweden \\ ${ }^{c}$ Dept Press, C15-1 TMNKM, Saab Automobile, SE-461 80 Trollhättan, Sweden \\ ${ }^{d}$ Department of Engineering Science, University West, SE-461 86 Trollhättan, Sweden
}

\begin{abstract}
In order to be able to form high strength steels with low ductility, multi-step forming processes are becoming more common. Benchmark 4 of the NUMISHEET 2011 conference is an attempt to imitate such a process. A DP780 steel sheet with $1.4 \mathrm{~mm}$ thickness is considered. In order to understand the pre-strain effect on subsequent forming and springback, a 2D draw-bending is considered. Two cases are studied: one without prestrain and one with $8 \%$ pre-stretching. The draw-bending model is identical to the "U-bend" problem of the NUMISHEET'93 conference. The purpose of the benchmark problem is to evaluate the capability of modern FEmethods to simulate the forming and springback of these kinds of problems.

The authors of this article have previously made exhaustive studies on material modeling in applications to sheet metal forming and springback problems, [1],[2],[3]. Models for kinematic hardening, anisotropic yield conditions, and elastic stiffness reduction have been investigated. Also procedures for material characterization have been studied. The material model that mainly has been used in the current study is based on the Banabic BBC2005 yield criterion, and a modified version of the Yoshida-Uemori model for cyclic hardening. This model, like a number of other models, has been implemented as User Subroutines in LS-DYNA. The effects of various aspects of material modeling will be demonstrated in connection to the current benchmark problems.

The provided material data for the current benchmark problem are not complete in all respects. In order to be able to perform the current simulations, the authors have been forced to introduce a few additional assumptions. The effects of these assumptions will also be discussed.
\end{abstract}

Keywords: Multi-stage forming, springback, material modeling, pre-straining

PACS: Replace this text with PACS numbers; choose from this list: http://www.aip.org/pacs/index.html

\section{INTRODUCTION}

Multi-step forming of sheet metal materials is a kind of process that is becoming more and more common in the industry. In terms of numerical simulations, very little has been done in this field. Benchmark 4 of the NUMISHEET 2011 conference is an attempt to imitate such a process. In order to understand the pre-strain effect on subsequent forming and springback, a 2D draw-bending is considered. Two cases are studied: one without pre-strain and one with $8 \%$ pre-stretching before the draw-bending process. The draw-bending model is identical to the "U-bend" problem of the NUMISHEET'93 conference. The purpose of the benchmark problem is to evaluate the capability of modern FE-methods to simulate the forming and springback of these kinds of problems.

The current paper starts with a brief description of the material models used in the present study. The following section describes the material parameter identification processes, and in the final section the analyses of the drawbending problem are accounted for. 


\section{MATERIAL MODELING}

The authors of the present paper have earlier shown the importance of the modeling of the material behavior for successful springback predictions [1],[2],[3]. In the previous work it is demonstrated that there are three material model constituents, which are important for a proper description of the material behavior. These are: the yield surface, the hardening law, and, finally, the modeling of the elastic stiffness degradation with plastic strain. Below follows a brief description on how these constituents are chosen for the present problem.

\section{Yield Surface}

To accurately account for the anisotropic behavior, the eight parameter yield criterion by Banabic and Aretz [6] is chosen:

$$
\bar{\sigma}=\left[\frac{1}{2}\left(|\Gamma+\Psi|^{M}+|\Gamma-\Psi|^{M}+|2 \Lambda|^{M}\right)\right]^{\frac{1}{M}}
$$

The functions $\Gamma, \Psi$ and $\Lambda$ are defined as

$$
\begin{aligned}
& \Gamma=\frac{L \sigma_{x x}+K \sigma_{y y}}{2} \\
& \Psi=\sqrt{\frac{\left(N \sigma_{x x}-P \sigma_{y y}\right)^{2}}{4}+Q^{2} \sigma_{x y} \sigma_{x y}} \\
& \Lambda=\sqrt{\frac{\left(R \sigma_{x x}-S \sigma_{y y}\right)^{2}}{4}+T^{2} \sigma_{x y} \sigma_{x y}}
\end{aligned}
$$

where the parameters $L, K, N, P, Q, R, S$ and $T$ are identified from uniaxial- and equibiaxial-test data.

\section{Kinematic Hardening Description}

To account for the material behavior at cyclic loading, two different kinematic hardening laws are considered: the classical hardening law by Arnstrong and Frederick (A-F) [7] and a modified version of the Yoshida-Uemori (Y$\mathrm{U})$ [8] hardening law. The back-stress evolution of the A-F law is described by:

$$
\dot{\boldsymbol{\alpha}}=C_{x}\left[\alpha_{s a t} \frac{\boldsymbol{\sigma}-\boldsymbol{\alpha}}{\bar{\sigma}}-\boldsymbol{\alpha}\right] \dot{\bar{\varepsilon}}^{p}
$$

where $C_{x}$ and $\alpha_{\text {sat }}$ are material parameters. The A-F hardening law is able to account for the Bauschinger effect and the transient behavior during reverse loading. However, the important permanent softening effect (see Refs. $[1][3][8])$ is not accounted for.

To also consider the permanent softening effect, a modified version of Y-U hardening law is also used in this study. In this modified version of the Y-U model, the plastic hardening curve is provided as input data, instead of being a part of the parameter identification procedure as in the original formulation. This also results in that the number of unknown material parameters is reduced from seven to four. Below follows a brief description of this modified version. For a full description the reader is referred to earlier work by the authors [1], [3]. The Y-U model includes both translation and expansion of the bounding surface, while the active yield surface only evolves kinematically. The evolution of the back-stress is expressed as:

$$
\dot{\boldsymbol{\alpha}}=\dot{\boldsymbol{\alpha}}^{*}+\dot{\boldsymbol{\beta}}
$$

with 


$$
\begin{aligned}
& \dot{\boldsymbol{\alpha}}^{*}=C_{x} \cdot\left(\frac{R}{Y} \cdot(\boldsymbol{\sigma}-\boldsymbol{\alpha})-\boldsymbol{\alpha}^{*}\right) \cdot \dot{\bar{\varepsilon}}_{p} \\
& \dot{\boldsymbol{\beta}}=k \cdot\left(\frac{b}{Y+R} \cdot(\boldsymbol{\sigma}-\boldsymbol{\beta})-\boldsymbol{\beta}\right) \cdot \dot{\bar{\varepsilon}}_{p}
\end{aligned}
$$

where $\boldsymbol{\alpha}^{*}$ is the relative kinematic motion of the yield surface with respect to the bounding surface, $\boldsymbol{\beta}$ is the centre of the bounding surface, $Y$ is the size of the yield surface, $C_{x}, b$ and $k$ are material parameters, and $R$ is the isotropic hardening of the bounding surface. In the current modified version, the isotropic hardening is described as:

$$
\dot{R}\left(\bar{\varepsilon}^{p}\right)=\dot{H}\left(\bar{\varepsilon}^{p}\right)-\dot{\beta}\left(\bar{\varepsilon}^{p}\right)
$$

where $H\left(\bar{\varepsilon}^{p}\right)$ is the given plastic hardening curve, and $\beta\left(\bar{\varepsilon}^{p}\right)$ is the uniaxial backstress of the bounding surface.

Besides the three already mentioned material parameters, the Y-U model also includes a fourth material parameter, $h$. This parameter affects the amount of cyclic hardening. The larger value of the parameter $h$ the smaller the prediction of cyclic hardening.

\section{Elastic stiffness}

The amount of springback during unloading depends to a great extent on the elastic stiffness of the material. It has been shown that during plastic deformation, the elastic stiffness decreases [1][3][9].

In this work, an analytical expression proposed by Yoshida et al. [8], was used to describe the elastic stiffness degradation with plastic strain:

$$
E_{u}=E_{0}-\left(E_{0}-E_{s a t}\right) \cdot\left(1-e^{-\xi \cdot \bar{\varepsilon}^{p}}\right)
$$

where $E_{0}$ is the initial Young's modulus, $E_{\text {sat }}$ is a value that the unloading modulus saturates towards, and $\xi$ is a material parameter.

\section{MATERIAL CHARACTERIZATION}

The material used in this study is a DP780 material with a thickness of $1.4 \mathrm{~mm}$. The material characterization is based on a number of experiments. Below follows a brief description of these experiments, and on how the various material parameters are determined from these experiments.

\section{Uniaxial Tension Tests}

Tension tests were performed in the rolling, transverse and diagonal directions. The basic experimental data from the tension test in the rolling direction are presented in TABLE 1. The uniaxial tension tests also form the basis for description of the anisotropic behavior of the material. Unfortunately, only the material coefficients for the Barlat Yld2000 and the Hill'48 yield criteria are provided in the benchmark material data sheet. However, the Banabic BBC2005 model (used in our simulations) and the YLD2000 model are identical, although their coefficients have different meanings. It has therefore been possible to transform the YLD2000 coefficients to BBC2005 coefficients. The recalculated coefficients for the BBC205 criterion are presented in TABLE 2. The exponent $M(a$ in Yld2000) has the same meaning for both criteria and is set to 6 .

TABLE 1. Uniaxial tension test data

\begin{tabular}{cccccc}
\hline Direction & E [GPa] & YS [MPa] & UTS [MPa] & R-value & Poisson's ratio \\
\hline Rolling direction & 198.8 & 527.0 & 831.5 & 0.781 & 0.3 (assumed) \\
\hline
\end{tabular}

TABLE 2. Material constants for the Banabic 8-parameter (BBC2005) yield criterion

\begin{tabular}{cccccccccc}
\hline Sample & $\boldsymbol{L}$ & $\boldsymbol{K}$ & $\boldsymbol{N}$ & $\boldsymbol{P}$ & $\boldsymbol{Q}$ & $\boldsymbol{R}$ & $\boldsymbol{S}$ & $\boldsymbol{T}$ & $\boldsymbol{M}$ \\
\hline DP780 & 1.029 & 0.925 & 1.043 & 1.062 & 1.032 & 0.956 & 0.995 & 1.004 & 6 \\
\hline
\end{tabular}


The uniaxial tension test in the rolling direction also forms the basis for the plastic hardening curve. However, due to the occurrence of diffuse necking, plastic hardening data is only provided up to $10 \%$ plastic strain. The maximum plastic strain level that can be anticipated in the springback examples is much higher than that. This means that the plastic hardening curve has to be extended up to higher values of effective plastic strain by extrapolation. This introduces a significant source of error in the simulations. It would have been desirable, if the experimental hardening curve was extended by means of data from a bulge test or a shear test.

In our simulations the hardening curve is extended by a simple extrapolation of Hollomon type:

$$
H\left(\bar{\varepsilon}_{p}\right)=K \cdot \bar{\varepsilon}_{p}^{n}
$$

where $H$ is the effective stress, and $K$ and $n$ are material parameters. Both these parameters are determined based on the slope at the end of the experimental tension test curve, such that $K=122.4$ and $n=0.125$. An illustration of the extrapolation is given in FIGURE 1a. The resulting hardening curve can be seen in FIGURE 1b, where the first part of the curve is the tension test data and the second part is based on the extrapolation described above. In the numerical simulations this hardening curve is given directly in the input data.
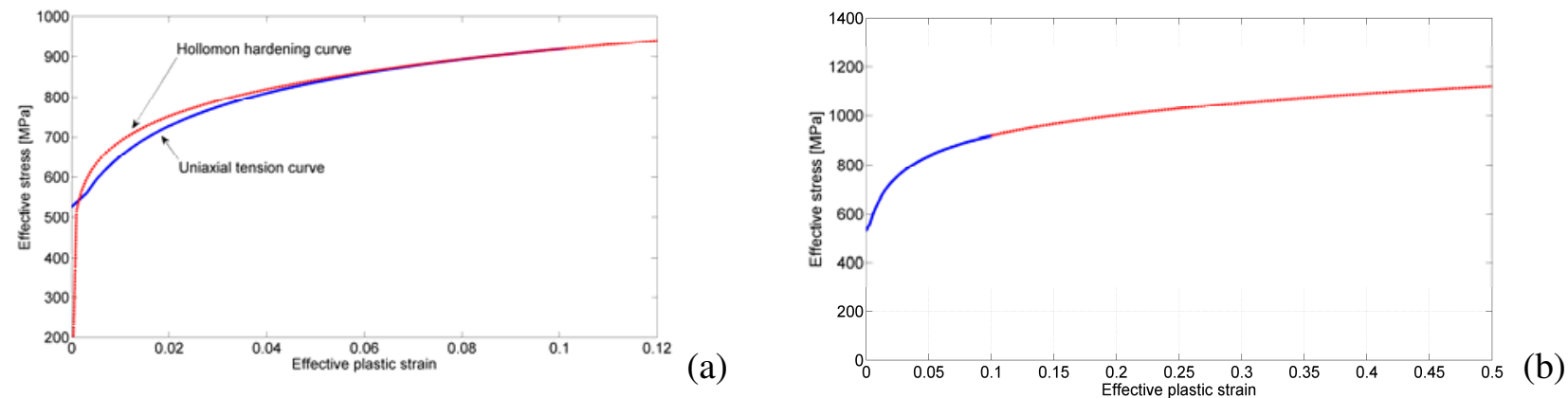

FIGURE 1. (a) Extrapolation of the isotropic hardening curve. (b) Resulting isotropic hardening curve with one part from uniaxial tension test in the rolling direction and one part from Hollomon extrapolation.

\section{In-Plane Tension-Compression-Tension Tests}

In order to characterize the cyclic behavior of the material, in-plane tension-compression-tension tests were performed. The test specimens were prepared in the rolling direction, using the testing apparatus described in Kuwabara et al. [4], for different amounts of pre-strains: 0.02, 0.04, 0.06, 0.08 and 0.1. The identification procedure is performed with the optimization code LS-OPT, such that a stress-strain relationship from the constitutive driver becomes as close to the experimental values as possible. In the optimization procedure we have chosen to use two of the experimental stress-strain curves as target curves: the curves corresponding to 0.04 and 0.1 plastic pre-strain. The reason for using two target curves is to assure that the obtained parameter set-up covers a wide range of plastic strain levels. By using only one target curve, there is a risk that the optimal solution only is suitable for that particular strain level. A detailed discussion on this matter can be found in an earlier work by the authors [5]. The obtained material parameters are provided in TABLE 3. In FIGURE 2 the performance of the kinematic hardening models with the obtained material parameters is shown.

TABLE 3. Kinematic hardening parameters for the two considered hardening laws

\begin{tabular}{cccccc}
\hline Hardening law & $\boldsymbol{C}_{\boldsymbol{x}}$ & $\boldsymbol{\alpha}_{\text {sat }}$ & $\boldsymbol{b}$ & $\boldsymbol{k}$ & $\boldsymbol{h}$ \\
\hline $\mathrm{A}-\mathrm{F}$ & 37.6 & 327.5 & - & - & - \\
\hline Y-U & 137.9 & - & 165.7 & 29.1 & 0.99 \\
\hline
\end{tabular}




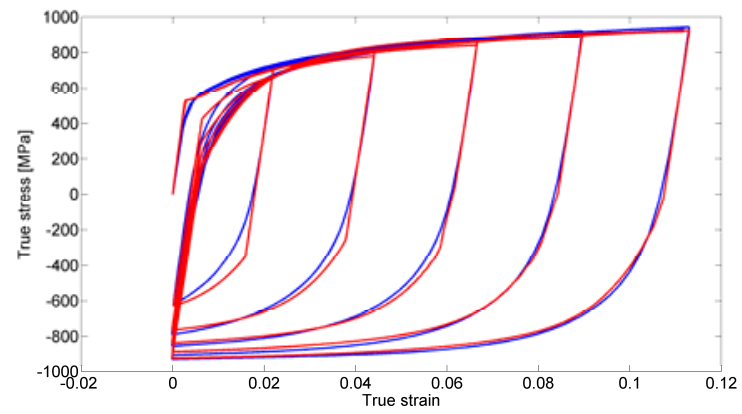

(a)

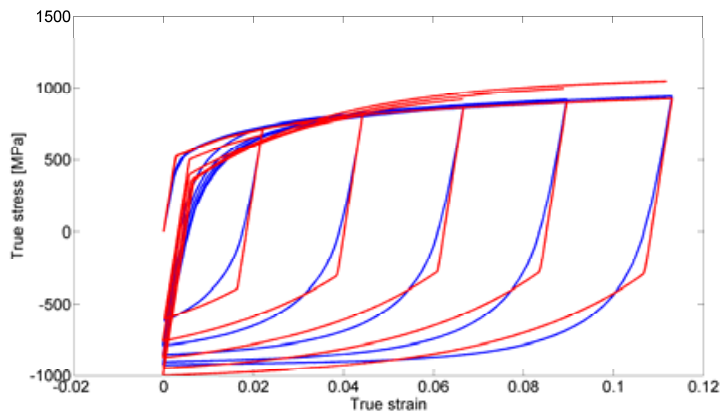

(b)

FIGURE 2. Verification of predicted uniaxial tension-compression-tension data for: (a) the modified Yoshida-Uemori kinematic hardening model and, (b) the Armstrong-Frederick hardening law, with material parameters according to TABLE 3. The red curves represent predicted values and the blue curves are experimental data.

\section{Elastic Stiffness Degradation}

The stiffness degradation as a function of plastic strain is measured from stress-strain relationships at five different levels of pre-straining. Based on this data, the material coefficients in Eq. 7 are determined by a least square fitting of the analytical expression to the experimental data points. In TABLE 4 the obtained parameters are listed. An illustration of the obtained analytical curve can be seen in FIGURE 3. In the figure the experimental data points are illustrated as red "stars".

TABLE 4. Material constants describing the elastic stiffness degradation according to Eq. 7

\begin{tabular}{lccc}
\hline Sample & $\boldsymbol{E}_{\boldsymbol{0}}$ [GPa] & $\boldsymbol{E}_{\text {sat }}[\mathbf{G P a}]$ & $\boldsymbol{\xi}$ \\
\hline DP780 & 198.8 & 167.0 & 87.0 \\
\hline
\end{tabular}

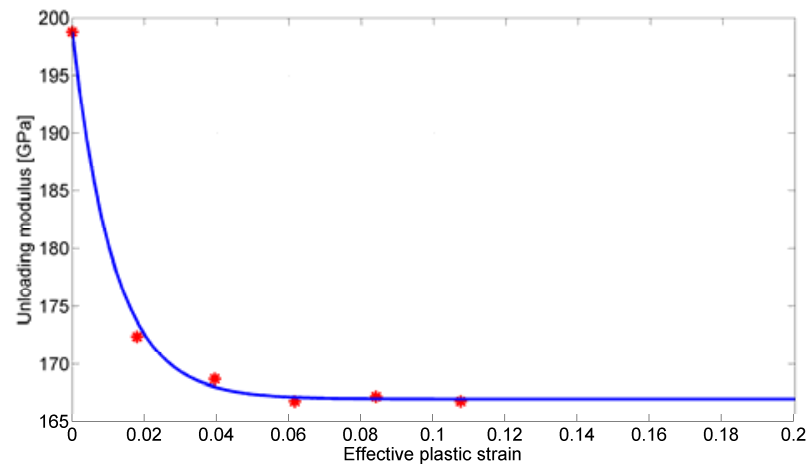

FIGURE 3. Unloading modulus curve according to Eq. 7 with parameters presented in TABLE 4 . The stars represents experimental data.

\section{SPRINGBACK}

The aim of this paper is to discuss benchmark problem 4 of this conference. The benchmark considers 2D-draw bending of the DP780-steel described earlier in this paper. Two different cases are compared: in the first one the virgin base material is used in the draw bending process, and in the second case the material is pre-tensioned up to $8 \%$ engineering strain. An illustration of the experimental set-up is given in FIGURE 4a, and the dimensions of the tools are provided in TABLE 5. The applied blank holding force is $2.94 \mathrm{kN}$. In FIGURE 6 and TABLE 7 the dimensions of the specimens used in the draw-bending example are defined.

\section{Numerical Simulations}

The numerical simulations were performed by means of the LS-DYNA code. For the forming simulations an explicit time integration scheme was used. The sheet material was modeled by fully integrated quadratic shell 
elements. The forming tools were modeled by shell elements and were assumed to be completely rigid. The friction coefficient between all contact surfaces was assumed to follow the simple Coulomb frictional law with a constant friction coefficient equal to 0.1 . The importance of the mesh size in springback simulations has been demonstrated in previous investigations. In this study a mesh convergence analysis was carried out. The result from the mesh sensitivity study is shown in FIGURE 5 and TABLE 6. As can be seen, the mesh that is called "mesh 3", $0.469 x 0.469$ $\mathrm{mm}$, seems to be the best choice. Therefore, this mesh density is used for both the considered cases.

The pre-straining of the material is modeled such that the nodes in one end of the material are locked in the length direction, while the nodes in the other end are given a prescribed displacement, corresponding to an axial strain level of $8 \%$ engineering strain. After the pre-straining, a springback simulation is performed so that the specimen becomes stress-free. The pre-strained specimen is then used in a forming operation, and a subsequent springback simulation.

For the springback simulations implicit time integration is used. The unloading method for the springback simulation is the so-called "one step unloading" method, which means that all the forming tools are removed before the springback calculation takes place.
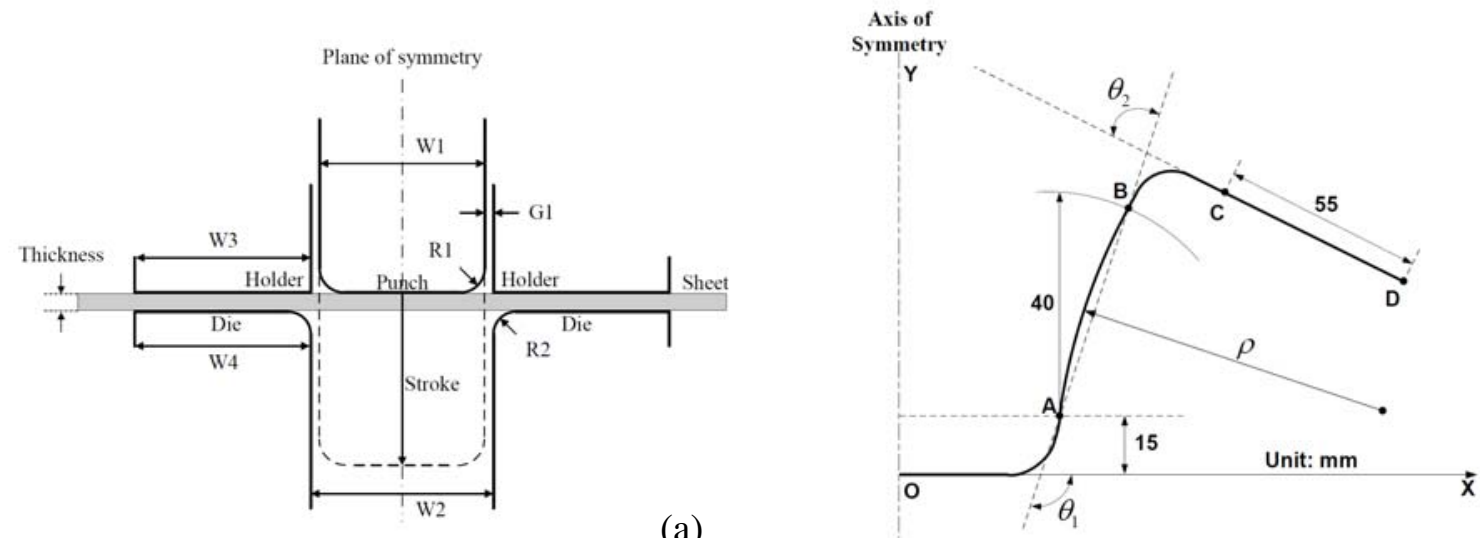

(a)

FIGURE 4. (a) Schematic view of the tools of the 2-D draw bending test. (b) Definition of the various springback measures.

TABLE 5. Dimensions for the 2D draw-bending test

\begin{tabular}{lllllllll}
\hline Parameters & W1 & W2 & W3 & W4 & R1 & R2 & G1 & Stroke \\
\hline Dimensions & 50.0 & 54.0 & 89.0 & 89.0 & 5.0 & 7.0 & 2.0 & 71.8 \\
\hline
\end{tabular}

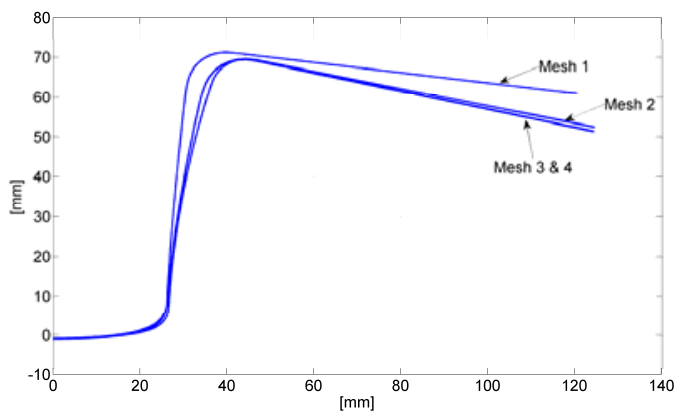

(a)

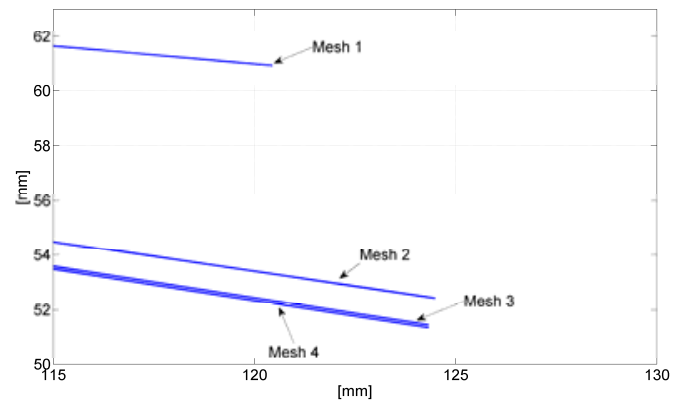

FIGURE 5: Illustration of the mesh sensitivity. (a) springback profile, (b) zoom-in of the specimen tips in figure (a). 
TABLE 6: Mesh-sizes in the mesh sensitivity study (FIGURE 5)

\begin{tabular}{ll}
\hline Mesh & Size $[\mathbf{m m ~ x ~ m m ]}$ \\
\hline Mesh 1 & $1.875 \times 1.875$ \\
\hline Mesh 2 & $0.938 \times 0.938$ \\
\hline Mesh 3 & $0.469 \times 0.469$ \\
\hline Mesh 4 & $0.234 \times 0.234$ \\
\hline
\end{tabular}

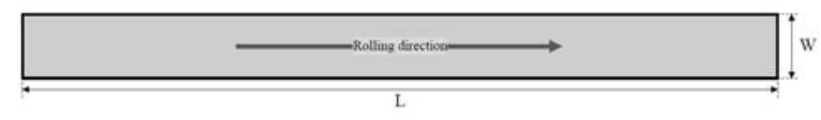

(a)

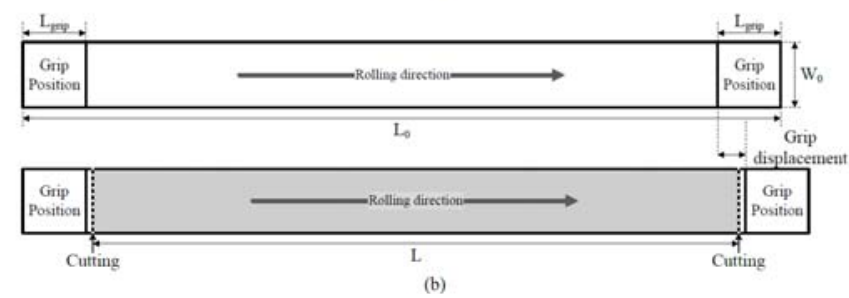

FIGURE 6. Schematic view of specimens and their dimensions for: (a) the base material case (without pre-strain), (b) the prestrained case.

TABLE 7. Dimensions of the specimens in FIGURE 6.

\begin{tabular}{cccccccc}
\hline & \multicolumn{3}{c}{ Base material } & \multicolumn{3}{c}{ Pre-strained specimen (8\% engineering strain) } \\
Parameters & $\mathbf{L}$ & $\mathbf{W}$ & $\mathbf{L}_{\mathbf{0}}$ & $\mathbf{W}_{\mathbf{0}}$ & $\mathbf{L}_{\text {grip }}$ & Grip displacement & $\mathbf{L}$ \\
\hline Dimensions & 360.0 & 30.0 & 360.0 & 30.0 & 25.0 & 24.3 & 324.0 \\
\hline
\end{tabular}

The springback profiles for the two considered kinematic hardening laws are displayed in FIGURE 7. FIGURE 7a shows the results for the base material, while in FIGURE $7 \mathrm{~b}$ the corresponding results for the pre-strained material are displayed. Obviously the A-F hardening model yields more springback than the Y-U model. The reason for this can be deduced from the determination of the kinematic hardening parameters, and from the stress-strain relationship for the A-F hardening law at high cyclic strain levels (see FIGURE $2 b$ ). As can be seen in FIGURE $2 b$ the predicted stresses at reverse loading are significantly higher than the experimental ones. This means that the numerical simulations of the draw-bend test, using the A-F hardening law, will also over-predict the stresses in the side-wall of the formed part. Therefore, also the bending moment will be over-predicted, and as a consequence of this also the resulting springback. Comparing FIGURE 7a and FIGURE 7b, we note that the difference between the results for the two considered hardening laws is larger for the base material case than for the pre-strained case

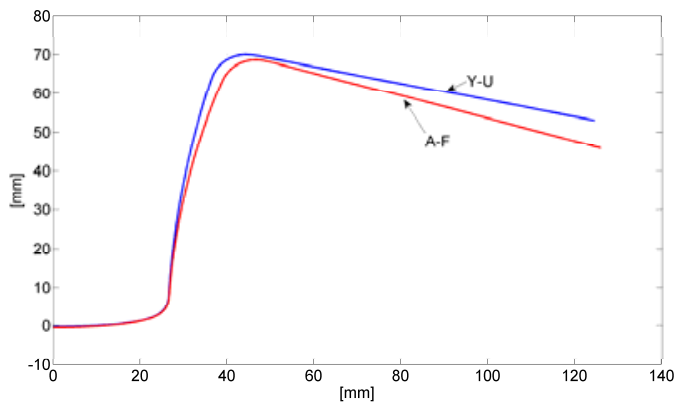

(a)

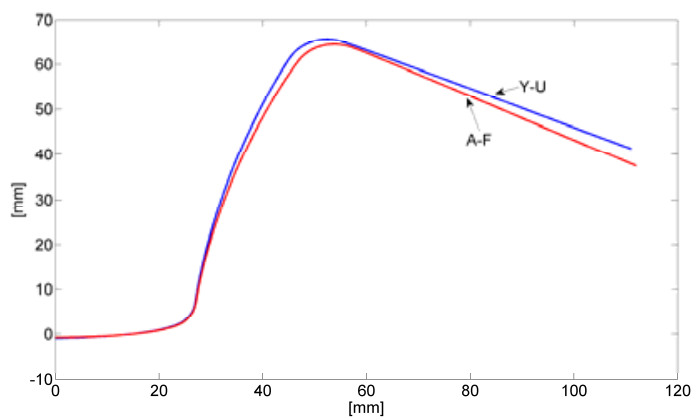

FIGURE 7. Springback profiles for (a) the base material, (b) the pre-strained material.

Since the Y-U hardening model fits experimental data best, it can be anticipated to result in the most accurate springback predictions. The resulting springback profiles for the Y-U model applied to the base material and to the pre-strained material, respectively, are shown in FIGURE 8a. It can be observed that the pre-strained material results in considerably more springback. The reason for this can be seen in FIGURE $8 \mathrm{~b}$. The figure shows the through 
thickness stress distribution at a point in the middle of the side wall, after the forming phase, and before the springback phase. As can be seen the stresses are much higher for the pre-strained case, which in turn results in higher side-wall curl. The reason for the higher stresses in the pre-strained case can easily be understood, if one considers the pre-strained material just as a material with a higher hardening curve than the base material.

In TABLE 8 the values of the springback measurements illustrated in FIGURE $4 \mathrm{~b}$ are shown for both the base material and for the pre-strained material, for the two considered kinematic hardening laws.
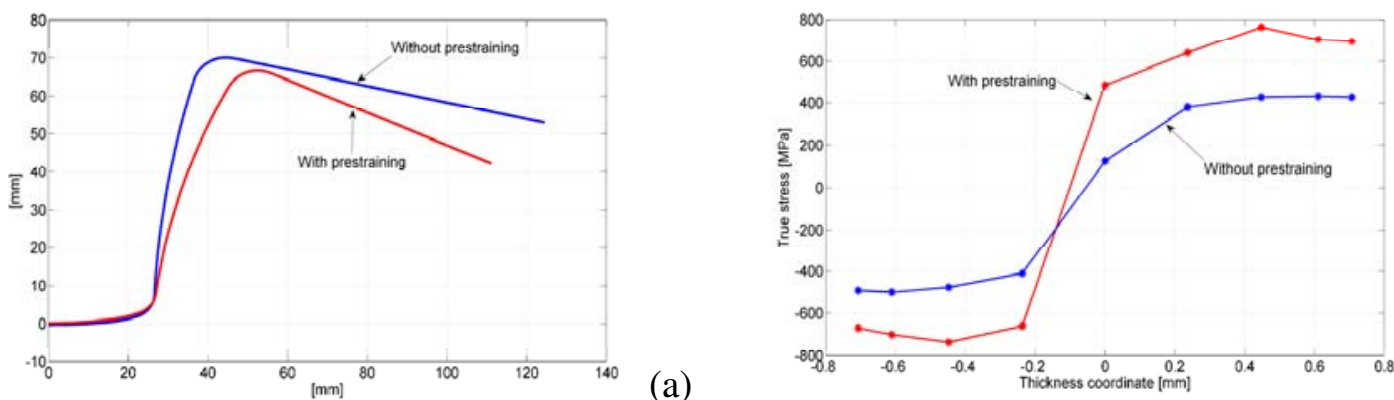

FIGURE 8. Y-U hardening model. (a) Springback profiles for the base material and the pre-strained material. (b) Longitudinal stress distribution through the thickness, for the base material, and for the pre-strained material, in a point in the middle of the sidewall.

TABLE 8. Springback measurements for all considered cases

\begin{tabular}{ccccccc}
\multicolumn{4}{c}{ TABLE 8. Springback measurements for all considered cases } \\
\hline Hardening law & $\boldsymbol{\theta}_{\boldsymbol{1}}$ & $\boldsymbol{\theta}_{\boldsymbol{2}}$ & $\boldsymbol{\rho}$ & $\boldsymbol{\theta}_{\boldsymbol{1}}$ & $\boldsymbol{\theta}_{\boldsymbol{2}}$ & $\boldsymbol{\rho}$ \\
\hline $\mathrm{Y}-\mathrm{U}$ & 97.2 & 81.5 & 655 & 105.5 & 79.5 & 525 \\
\hline $\mathrm{A}-\mathrm{F}$ & 99.4 & 80.0 & 542 & 107.2 & 78.0 & 466 \\
\hline
\end{tabular}

\section{CONCLUSIONS}

Benchmark 4 of the NUMISHEET 2011 conference has been studied. It has been shown that the springback deformation after a draw-bend operation, such as the one presented in the benchmark, is very sensitive to the mesh size and the choice of kinematic hardening model. In this paper both the A-F hardening law and the Y-U hardening law has been used. Since the Y-U hardening model fits experimental cyclic stress-strain data best, it can be anticipated to yield the most accurate springback predictions.

When it comes to the effect of pre-straining of the material before the draw-bend operation, it is shown that the resulting springback for the pre-strained case becomes higher than for the base material case. This is a result of the prediction of higher stresses in the side-wall after the forming stage, resulting in larger side-wall curl, for this case.

It should finally once again be pointed out that the numerical results obtained in this study are highly dependent on the appearance of the hardening curve, which we, unfortunately, to a great extent have had to guess. The errors originating from this assumption may perhaps be of the same order as the differences in results obtained with the two kinematic hardening laws.

\section{REFERENCES}

1. P.A. Eggertsen and K. Mattiasson, Int. J. of Mech. Sci. 51, 547-563 (2009).

2. P.A. Eggertsen and K. Mattiasson, Int. J. of Mech. Sci. 52, 804-818 (2010).

3. P.A Eggertsen, "Prediction of springback in sheet metal forming - with emphasis on material modeling", licentiate Thesis, Chalmers University of Technology, 2009.

4. Kuwabara et al, Int. J. of Plast.. 25, 1759-1776 (2009).

5. P.A. Eggertsen and K. Mattiasson, Int. J. of Mtrl. Forming (2011).

6. D. Banabic et al, Int. J. of Plast, 21, 493-512 (2005).

7. P.J. Armstrong and C.O. Frederick, G.E.G.B report RD/B/N 731 (1966).

8. F. Yoshida and T. Uemori, Int. J. of Plast, 18, 661-686 (2002). 